\title{
Il Castello di "Carta". Excursus della presenza del castello di Gallipoli nella cartografia storica
}

The Castle of "paper". Excursus of Gallipoli's castle presence in historical cartography

\section{Aurora Quarta}

Università del Salento, Lecce, Italy, aurora.quarta@gmail.com

\begin{abstract}
The castle is located at the eastern part of the Gallipoli's old town: the first data in archives and libraries started from the sixth century under the mention of castrum and in the following centurie there are many informations on parchments, written documents and bibliography published until today. The Syllabus Grecarum Membranarum from the twelfth century and the Statutum de reparatione castrorum of Frederick II are two precious sources about the primitive castle's architecture.

The structure endured the passage of the Byzantines, Normans, Swabians, Angevins and again, Aragonese, Venetians, Spaniards, Austrians and finally the Bourbons, until it became property of the State and now of the Gallipoli's municipality. It has suffered over time numerous interventions to adapt it to new military needs: the castle was no longer effective with leading defense from new siege weapons, as for other architectures of the same period.

The numerous representations preserved in Italian and European archives give a complete picture of the Gallipoli's urban development and include the defensive system of the city: the different views illustrate the walls and allow us to understand the castle's main evolutionary dynamics and its connection with the town.
\end{abstract}

Keywords: Gallipoli, carthography, castle.

\section{Introduzione}

Gallipoli ha rivestito, all'interno del Mediterraneo, nel corso dei secoli, un ruolo importante (Fig. 1): attualmente, il suo sistema difensivo, di cui il primo impianto non è tutt' ora noto, si conserva in maniera quasi integrale. Numerose sono le pubblicazioni che trattano e menzionano la struttura, nella maggior parte dei casi riproponendo le medesime informazioni relative alla sua origine, i suoi cambiamenti, miglioramenti e modifiche; analogamente, ricca è la documentazione d'archivio le cui prime menzioni risalgono al VI secolo con un castrum della città, ed al 1195 con una turris civitatis (Hartmann, Ewald, 1957, p. 193; Trinchera, 1865, p. 520). Nel complesso, il castello di Gallipoli è parte integrante della fortificazione gallipolina assieme ai $12 \mathrm{ca}-$ pisaldi della cinta muraria (Fig. 2), presenta una pianta quadrilatera, come molti altri castelli in Terra d'Otranto, sui cui spigoli vi erano un tempo le quattro torri attualmente ridottesi a tre per un cedimento strutturale che ha portato al crollo di una di esse. Ancora visibile è il torrione poligonale e le rondelle mentre, è affiancato sul lato orientale dalla struttura del Rivellino, porzione della struttura aggiunta nel XVI secolo e, successivamente, divisa dal castello (Quarta, 2017b, p. 92).

L'importanza della città è confermata dalla quantità di documentazione scritta conservatasi 
nel tempo alla quale si sommano le numerose rappresentazioni nella cartografia sparsa in diversi archivi italiani e stranieri, edita e non, datata dal XVI al XIX secolo, per la maggior parte dei casi.

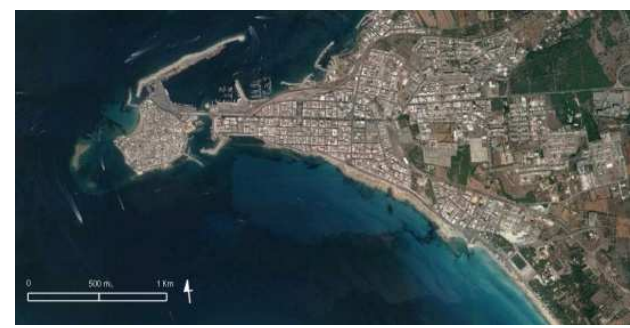

Fig. 1. Immagine satellitare della città di Gallipoli (Google Earth).

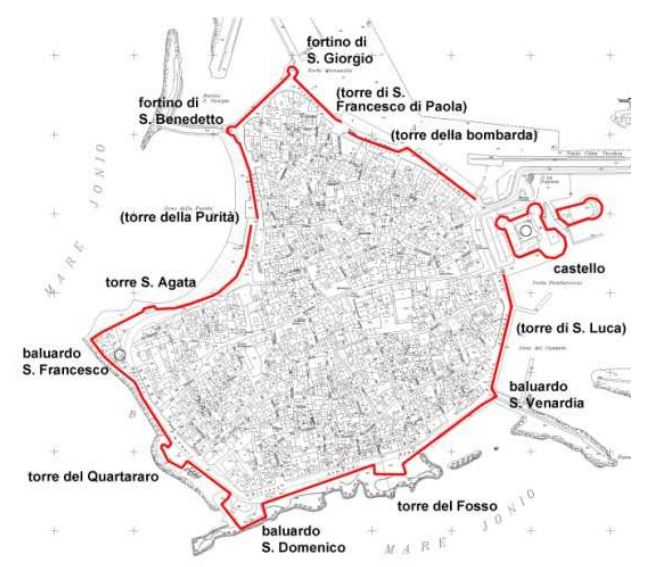

Fig. 2. Sistema difensivo di Gallipoli (Quarta 2016).

\section{Il castello come "palinsesto dell'archi- tettura militare"}

Già in età bizantina sorge l'esigenza di “incastellarsi" dotandosi di strutture difensive che vedranno ulteriori interventi anche nel periodo normanno (Licinio, 2010, pp. 24-32). Il 1071 infatti, segna la fine dell'Impero Romano d'Oriente in Italia Meridionale anche se, già in precedenza si ha traccia del popolo normanno (Fuzio, 1981, p. 128): questi, una volta ottenuto il potere, lo parcellizzarono per mezzo di una suddivisione del territorio e solo successivamente procedettero ad una riorganizzazione a livello amministrativo (Poso, 1988, p. 37). L'incastellamento normanno ha portato alla co- struzione di diverse strutture in Terra d'Otranto edificate ex novo (Licinio 2010, p. 56; Willemsen - Odenthal 1966, p. 54) che configurano diverse linee difensive: quella interna con i castelli di Ceglie Messapica, Oria, Torre S. Susanna, quella adriatica arretrata rispetto la costa (Ostuni, San Vito dei Normanni, Mesagne, San Pietro Vernotico), quella ionica settentrionale collegata a Gioia del Colle (Massafra, Taranto, Lizzano) e quella ionica meridionale (Nardò, Fulcignano Galatone, Acquarica del Capo, Presicce) con Gallipoli come avamposto (Fuzio, 1981, p. 134) nel quale si ipotizza come traccia bizantina il torrione pentagonale (o esagonale) inglobato nelle fasi successive (Fuzio, 1981, p. 147; Licinio, 2010, p. 45). Le strutture normanne, come anche quelle sveve prima e angioine poi, si svilupparono in altezza per poter applicare il sistema difensivo del "tiro piombante" (Cariddi, 2014 , p. 20). Durante il periodo svevo, vengono rafforzati quasi tutti i castelli fulcro di una nuova ed organica struttura militare della regione ma, la fase sveva, come del resto le precedenti, non sempre risulta documentabile direttamente sugli edifici a seguito dei numerosi e successivi rimaneggiamenti che si sono susseguiti (Fuzio, 1981, p. 164). Analogamente, anche la documentazione scritta non risulta essere particolarmente ricca, almeno sino al 1220 quando il Regno di Sicilia passò a Federico II (Sthamer, 1995, p. 3). Con Federico II non si ebbero più le concessioni dei castelli ai feudatari bensì le deleghe con l'obbligo di amministrarli. Fondamentale è lo Statutum de reparatione castrorum (1241-1246) (Houben, 1996, p. 169), nelle cui annotazioni compare Gallipoli, citata da Federico II che ordinava "Castrum Galipuli debet reparari per barones Neritoni abbatem, Neritonecum feudo Soleti et Ogenti et ecclesie Ogenti habent es feuda et casalia eiusdem terre. Et homines Galipuli possunt reparare castrum cum predictis" (Sthamer, 1995, p. 106). Si deduce che la fortezza venne riparata non solo grazie agli abitanti, bensì anche attraverso l'intervento dei baroni e dell'abate di Nardò, l'ausilio della chiesa di Ugento e del feudo di Soleto e di Ugento.

Le opere castellari possono essere classificate in merito alle funzioni e usi che esercitavano, distinguendo il castello-rocca dal castello- 
residenza; analogamente, anche l'ubicazione può dare informazioni relative alla funzione e tra le tante, nel caso di Gallipoli, il castello è ubicato sulla costa dove controlla un insediamento urbano, un porto commerciale e un tratto di litorale: Gallipoli assieme a Otranto, Brindisi e Taranto, in Terra d'Otranto è tra i principali insediamenti portuali fortificati (Licinio, 2010, p. 138). Nel 1266 il regno passa a Carlo I d'Angiò che conferma la validità del sistema amministrativo svevo: nonostante ciò, proprio nei centri costieri sono concentrati i maggiori interessi ed ovviamente, le tensioni alimentate dalle mire espansionistiche angioine, dal movimento dei pellegrini in Terra Santa e, soprattutto, dai traffici commerciali assieme alle sempre presenti incursioni dal mare (Licinio, 2010, p. 168). Durante la fase angioina, i castelli vengono arricchiti da ulteriori elementi come la residenza temporanea del sovrano e la sede carceraria permanente, oltre ad essere potenziati per mezzo di presidi, depositi di armi e sedi per le guarnigioni (Licinio, 2010, p. 169; Schettini, 1959, p. 119). L'architettura militare subisce un'obbligata evoluzione come risposta ai cambiamenti in materia di difesa bellica: con l'apparizione delle armi da fuoco infatti, si introduce un nuovo elemento di difesa ed offesa che portò inizialmente ad un periodo di transizione in cui le vecchie fortificazioni furono in qualche modo riadattate e, in seguito, alla scomparsa delle parti meno resistenti a favore di nuovi sistemi di offesa (Petrignani, 1959, pp. 127-128). Nel caso di Gallipoli, se in un momento iniziale non si sente l'esigenza di intervenire sulla struttura, successivamente si opta per un adeguamento con diverse operazioni. Con il nuovo sovrano Alfonso d'Aragona si ottiene una "stabilizzazione dinastica" e una forte ripresa dell'incastellamento di borghi, casali, strutture monastiche e città (Licinio, 2000, p.301-302; Fuzio, 1981, p. 176) ma, soprattutto, due obiettivi: il consolidamento delle posizioni e l'intensificarsi della spinta verso l'Oriente (Carducci, 1995, p. 102).

La Terra d'Otranto, divenuta il punto più a rischio di attacchi a causa della sua posizione nel Mediterraneo, obbliga il Sovrano aragonese a fortificare la regione ed intervenire sulle fortezze (Pascalis, 2004, p. 162): l'evoluzione delle armi comporta l'adeguamento a livello architettonico e, infatti, a Gallipoli il castellano Diomede de Tana controlla i lavori presso il castello e l'edificazione delle casematte (1487). Successivamente alle restituzioni da parte dei veneziani delle città costiere occupate durante la guerra, il regno di Spagna si protrae per circa due secoli: nel 1503, la Spagna conquista il Regno di Napoli con Consalvo di Cordova e decide, ovviamente, di occuparsi del sistema difensivo costiero con riadeguamenti delle fortificazioni militari che risultarono obbligati. A dare una forte spinta agli interventi sulle fortificazioni, don Pedro de Toledo, Vicerè dal 1532 al 1553 particolarmente devoto a Carlo V che, seguito dal figlio Filippo II, approva l'opera riformatrice del Consiglio di Stato (Mauro, 1998, p. 53). Negli anni seguenti, le strutture vengono migliorate, ammodernate e adeguate anche con artiglieria e munizioni. La difesa del Regno, grazie ad un funzionale sistema militare costiero, risulta anche in seguito di fondamentale importanza, sia con Filippo III che incita a vigilare sulla costa del Regno, sia con $i$ suoi successori che si impegnano nei secoli successivi sulla manutenzione degli edifici. Nel 1707, il reame di Napoli passa agli austriaci mentre, nel 1734 il Castello passa ai Borbone fino al 1857 quando dal Governo borbonico viene radiato dal novero delle Piazze forti del Reame.

\section{Gallipoli nella cartografia storica}

L'ubicazione di Gallipoli sulla costa ionica della penisola salentina ha favorito nei secoli l'incremento commerciale del porto gallipolino ma, nello stesso tempo, ha avuto bisogno del continuo riammodernamento delle difese. L'abitato si è sviluppato su uno scoglio circondato da isolotti collegato alla terraferma da un ponte da cui sono ben evidenti, nelle rappresentazioni storiche, le diramazioni delle vie che fiancheggiavano i due litorali, inoltrandosi verso l'entroterra a collegamento degli centri urbani vicini. Tra le prime e più complete iconografie che rappresentano la città vi è un'incisione di Natale Bonifacio, commentata dal Crispo per l'editore fiammingo Nicola Van Aelst, divenuta in seguito la principale riproduzione planimetrico-topografica della città (Fig. 3). 


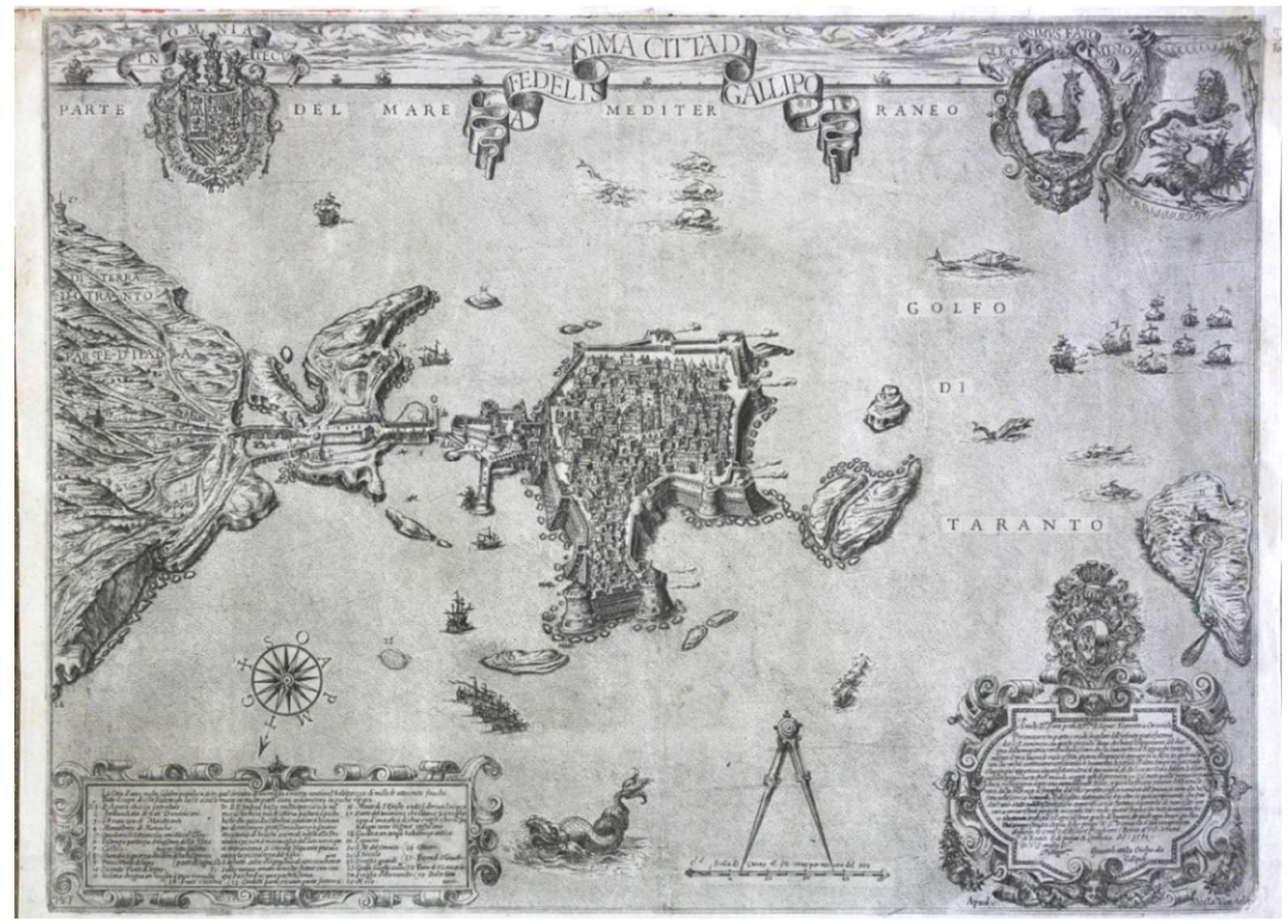

Fig. 3. "Rarissima pianta prospettica della città", 1591, incisione, Natale Bonifacio (Cazzato 1989).

Il nome in alto è affiancato dallo stemma di Filippo II e a destra da quello civico con il primo motto, Nec animus fato minor; in basso a sinistra, un cartiglio in cui si legge "la Citta d'aere molto salubre populosa, e in quel circuito di un miglio e mezzo contiene l'habitanzza di mille, e ottocento fuochi. Tutto il corpo di essa siede in un sasso a cui le mura in molte parti sono ornamento in poche riparo". Nello stesso cartiglio, son inseriti 29 numeri di riferimento alla cartografia stessa mentre, in quello ubicato a destra vi è un riferimento alle origini della città, al tempo riferite al mondo greco, attualmente di gran lunga superate (Ferrari, Scardozzi, 2016, pp. 1820).

È evidente l'alta urbanizzazione del borgo che sarà, poi, definito un "meandro inestricabile" (Giorgi, 1886, p. 56) e la cui caratteristica è già qui ben espressa. Il dettaglio dell'incisione non trascura il sistema difensivo che viene rappresentato in maniera puntuale: è possibile osservare in ogni torrione della cinta muraria tre livelli di bocche di fuoco e le merlature terminali, ana- logamente alle cortine delle mura evidentemente più alte rispetto alla situazione attuale. Nelle rappresentazioni storiche sono già ben visibili le fasi antecedenti agli adeguamenti del sistema di difesa, come nel caso del Fortino della Madonna degli Angeli, o del Ceraro, detta anche Torre del Governatore, o Torre del Quartararo, attualmente di forma poligonale (Fig. 2) ma con una planimetria circolare (Fig. 4) nelle carte antiche. L'incisione di Braun-Hogenberg (1598) raccolta nel Civitates Orbis Terrarum, quella di Jodocus Hondius del 1627 e quella del tutto simile nell'Isolario del Coronelli di fine secolo (Fig. 5), sono alcune delle numerose rappresentazioni attualmente note con chiaro riferimento all'incisione di Bonifacio.

Queste si differenziano dalle rappresentazioni del Bertelli (1629), del Merian (Fig. 6) e dall'incisione edita dall'Albrizzi (Fig. 7), altro gruppo di iconografie a cui somma quella dell'Adlerhold (1702) e di Blaeu (1663), le quali mostrano un abitato ben urbanizzato ma allo 
stesso tempo con una regolarità nell'impianto che distingue chiaramente gli isolati e le strade.
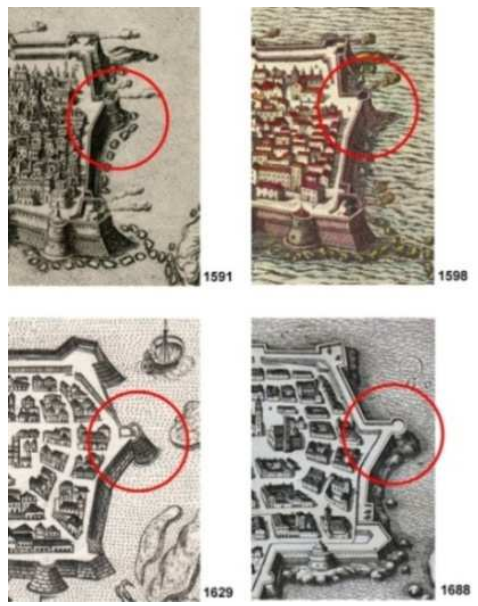

Fig. 4. Torre del Quartararo in diverse iconografie storiche (Quarta 2016).

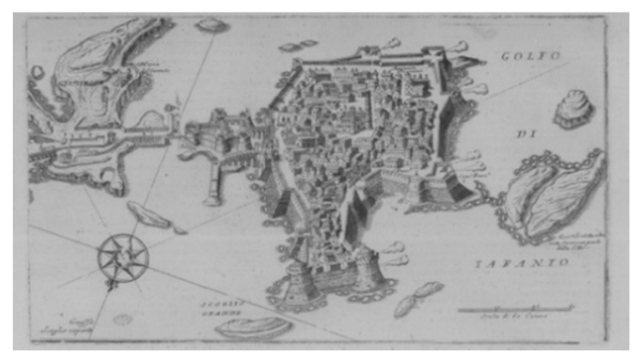

Fig. 5. Veduta di Gallipoli, incisione, 1696, in V.M. Coronelli, "Città e fortezze e porti principali dell'Europa" (Cazzato 1989).

Nell'abitato è evidente l'assenza di uno sviluppo rettilineo delle strade ed una mancanza di piazze (ad esclusione della piazza della Cattedrale) come racconta il Galateo: "Nulla viarum praecipue quae procedat ad Austro ad Boream, qui saepissime et vehementissime flaresolent, rectamfecit, sed alteri altera saepe occurrit: altera alteram sub inde intercipit, inflectitur, curvatur in gyrum, discurrit, redit in seipsam, nunc in rectum, nunc in obliquum solvitur angulum: nuncrecte, nunc oblique procedit, adeo ut antiquis habitatoribus difficiles sint viarum perplexi, et inxplicabiles, et amphractuosi meatus" (Paone, 1974, p. 242). Osservando tali rappresentazioni e confrontandole con lo stato attuale, è evidente come la città abbia conservato nel tempo il suo impianto, nonostante abbia poi accolto tutte le strutture successive (basti pensare ai numerosi palazzi nobiliari o agli edifici ecclesiastici barocchi). Un impianto urbano prospettato in questo modo era ed è, ancora oggi, un'ottima protezione dalle intemperie; se si accede da qualunque strada che lungo le mura, per inserirsi nel centro storico, si nota come essa, deviando quasi subito, ripari dagli agenti atmosferici.

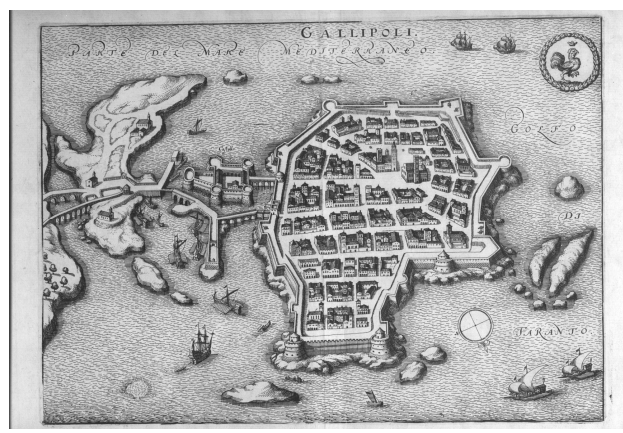

Fig. 6. Veduta di Gallipoli, 1688, incisione, in M. Merian, "Tophographia Italiae Das ist Warafte und Curiose Beschreibung Italien Sambt Einen Anhang von Königreich Morea", ristampa dal 1640. (http://www.fondazioneterradotranto.it/2015/11/14/gal lipoli-in-nove-mappe-antiche/).

Nel reticolo di strade, alcuni studiosi hanno intravisto alcune caratteristiche proprie dell'influenza islamica, la quale accomuna numerose città della Puglia, come Bitonto, Martina Franca, Bitetto, Altamura, Putignano, Grottaglie, per citarne alcune. La rete stradale ad andamento radiale viene schematizzata in tre modelli principali: lo shari, la via pubblica principale, il darb, la via secondaria che giunge nei diversi quartieri, e l'azucak, il vicolo cieco che giunge fino all'abitazione (Guidoni, 1991, p. 102). Non sarebbe da escludere, in realtà, un assetto urbanistico del centro storico come risultato di uno sviluppo dell'abitato spontaneo, libero e privo di una pianificazione programmata, dall'origine del primo impianto fino ad oggi. Il tessuto urbano ha subito diverse trasformazioni, ampliamenti, fusioni di vicoli ciechi, abbattimenti di edifici per la creazione di nuovi spazi col fine di ospitare strutture di maggiori dimensioni (Saladini, 1980, p. 350). 


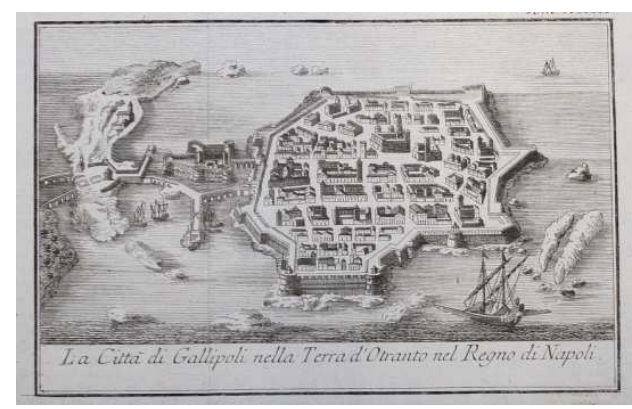

Fig. 7. Veduta di Gallipoli, 1761, incisione, in G. Albrizzi (http://www.fondazioneterradotranto.it/2015/ 11/14/gallipoli-in-nove-mappe-antiche/).

Nel secondo gruppo di rappresentazioni della città su citate, un minor dettaglio viene dato alla caratterizzazione del sistema di difesa anche se conserva le analoghe peculiarità delle precedenti menzionate. Differente è la planimetria della città conservata in Spagna nella raccolta del cardinale Antonio Perrenot de Granvelle (1517-1586) che raccoglie numerose città italiane e annesse fortezze (Brunetti, 2007, pp. 3-21), dove compare essenzialmente il sistema difensivo costituito dalla cinta muraria con torrioni e bastioni e dal castello (Fig. 8).

La torre del Governatore compare con planimetria circolare ma in trasparenza: la cortina che congiunge i baluardi di S. Francesco e di S. Domenico è continua e in bruno come nella restante difesa della città. In una corretta lettura e interpretazione di questo particolare, più che un'aggiunta di tale torrione, già attestato nella precedente documentazione, risulterebbe veritiera l'ipotesi di una progettazione al fine di eliminare la porzione che non avrebbe comportato un indebolimento, poiché la cortina muraria sarebbe stata difesa dal fuoco amico dei bastioni (Fig. 9).

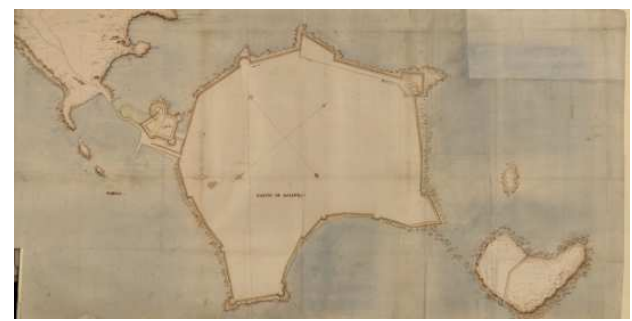

Fig. 8. Sistema difensivo di Gallipoli, XVI secolo, disegno acquerellato. () Patrimonio Nacional.

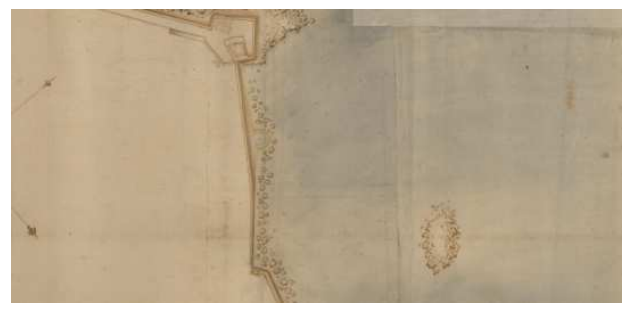

Fig. 9. Particolare della cinta muraria sul fronte ovest, XVI secolo. (C) Patrimonio Nacional.

\section{Il castello}

Il castello, nella sua attuale articolazione (Fig. 10), è il risultato di una lunga serie di aggiunzioni e rimaneggiamenti susseguitisi nei secoli per continue esigenze belliche (Vita, 1974, pp. 194195; Quarta, 2017b, p. 93). La struttura è parte fondamentale del sistema di difesa, ha planimetria quadrilatera sui cui spigoli sono inserite le rondelle, di cui a base poligonale quella di sudest, mentre a sud-ovest un crollo del XVIII secolo ha fatto sì che la rondella non giungesse sino ai nostri giorni (Quarta, 2017a, p. 149). Come per numerosi altri castelli, i torrioni sono estremamente sporgenti e con mura scarpate, un coronamento di archetti e beccatelli e diverse bocche di fuoco (Fig. 11).

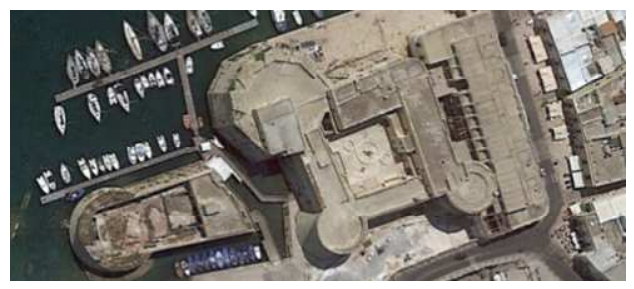

Fig. 10. Il castello visto dall'alto (Google Earth).

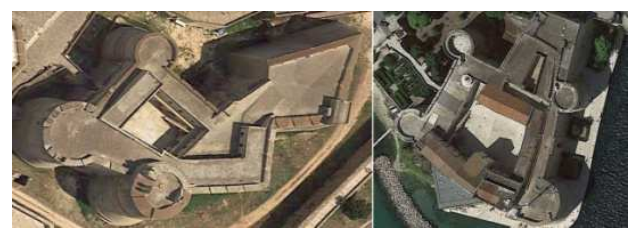

Fig. 11. I castelli di Otranto e Taranto in cui è visibile il rapporto tra rondelle e cortine murarie (Google Earth).

Il dettaglio raggiunto nelle vedute della città di Gallipoli, menzionate e non, permette di avere anche una precisa raffigurazione dell'edificio: in 
Valesio (1579), ad esempio, è visibile la sua planimetria quadrangolare, il prolungamento del Rivellino è fiancheggiato dal ponte che congiunge l'isola alla terraferma mentre, il castello si connette alla città per mezzo del ponte dell'Avanzata (Fig. 12).

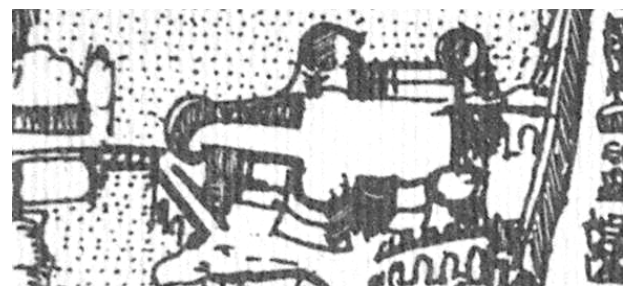

Fig. 12. Particolare del castello, 1579, incisione, in F. Valesio (Cazzato 1989).

Nulla permette di delineare più chiaramente l'articolazione della struttura come, di contro, accade nell'incisione di Bonifacio e nelle raffigurazioni successive che si ispirano ad essa (Fig. 5). In questi casi, risultano ben evidenti le rondelle con relativi tori marcapiani, l'articolazione interna della piazza d'armi, le aperture e bocche di fuoco sulle cortine del Rivellino, l'artiglieria pesante sul livello superiore e, inoltre, il ponte levatoio all'ingresso della città. La planimetria conservata a Madrid è, invece, la migliore testimonianza del castello alla fine del XVI (Fig. 13). Essa ci restituisce il primo livello dove compare chiaramente l'accesso dal ponte dell'avanzata con la chiara tipologia "a baionetta", gli ambienti che si affacciavano nella piazza d'armi ma, soprattutto, il punto preciso in cui le cortine del Rivellino si appoggiano al torrione poligonale ed al torrione della bandiera (Quarta, 2016, p. 94).

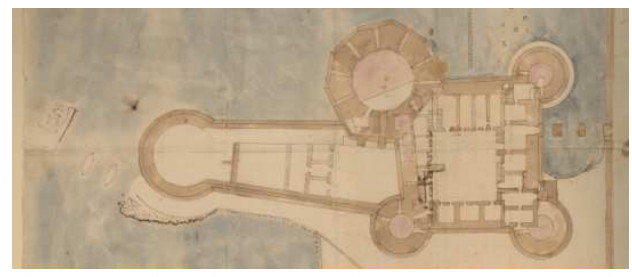

Fig. 13. Planimetria del castello, XVI secolo. (C) Patrimonio Nacional.

Osservando, poi, il particolare del castello nella veduta della città del Palacio Real di Madrid
(Fig. 8), il Rivellino compare in trasparenza come nel caso della torre del Governatore (Fig. 9): analogamente, sarebbe da ipotizzare una rinnovata progettazione della porzione Est del castello che avrebbe previsto l'eliminazione del puntone assieme alla Cortina orientale e, con tutta probabilità almeno in parte, del torrione della bandie$\mathrm{ra}$, e realizzare un nuovo bastione ben rimarcato nel disegno (Fig. 14).

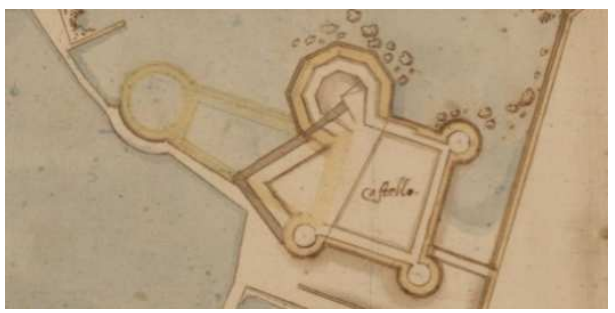

Fig. 14. Particolare della planimetria della città, XVI secolo. () Patrimonio Nacional.

Altro elemento da tenere in considerazione è l'ingresso al castello che, mentre nello stato attuale ed anche nella planimetria della raccolta di Perrenot (Fig. 13) è ubicato lungo il prospetto ovest ma nei pressi del torrione della campana, nel particolare tratto dal disegno dell'intero sistema di difesa (Fig. 14) l'ingresso è a ridosso del torrione opposto. Se si osserva attualmente la struttura, è possibile notare come in quel punto vi è un avancorpo quadrangolare che conserva al suo interno un piccolo ambiente suddiviso dall'adiacente per mezzo di un elegante arco. Tale avancorpo, quindi, compare nella planimetria di dettaglio del castello ma non nella pianta generale del sistema di difesa: sarebbe da ipotizzare, quindi, che le due si susseguono in tale ordine e che la variante del progetto dell'abbattimento del Rivellino (come anche del Torrione del Governatore) non è stata portata a compimento (in tal caso la planimetria di Perrenot potrebbe essere solo un progetto non eseguito), mentre risulterebbe verosimile lo spostamento dell'ingresso del castello, con un nuovo ponte dell'avanzata, lungo il prospetto occidentale da nord a sud.

\section{Conclusioni}

Lo studio sistematico del sistema di difesa della città, con particolare interesse verso la struttura 
del castello, attualmente in corso, sta restituendo notevoli dati e inaspettate sorprese. Il progetto è stato avviato nel $2014 \mathrm{col}$ fine di incrementare la conoscenza storico-architettonica dell'apparato difensivo contestualizzandola nell'ambito dell'architettura militare del Regno: i risultati at- tesi andranno sicuramente ad incrementare le limitate conoscenze del complesso gallipolino e da ciò, è lecito aspettarsi interessanti ricadute a livello scientifico legate alle variegate dinamiche costruttive individuate.

\section{Bibliography}

Bertelli, F. (1629). Theatro delle città d'Italia, Padova.

Brunetti, O. (2007). "Disegni di architetture militari nel Viceregno di Napoli dalla raccolta del cardinale Antonio Perrenot de Granvelle (1517-1786)", Kronos, 11, pp. 3-21.

Carducci, G. (1995). "La ricostruzione del castello di Taranto nella strategia difensiva aragonese (1487-1492)", ASP, XLVIII, pp. 101-178.

Cariddi, P. (2014). Otranto intra moenia. Dagli Aragonesi ad oggi, Manduria.

Cazzato, V. (1989). Atlante storico della Puglia. 4. La Provncia di Lecce, Cavallino.

Ferrari, I.; Scardozzi, G. (2016). Contributo alla carta archeologica di Alezio, Bari.

Ferrariis Galateo, A. De. (1974). Epistole Salentine (Ad Loysuim Palatinum - de Situ Iapygiae-Callipolis descriptio), Galatina.

Fuzio, G. (1981). "Castelli: tipologie e strutture”, C. D. Fonseca (a cura di), La Puglia tra Medioevo ed Età Moderna. Città e campagna, Milano, pp. 118-192.

Giorgi, C. De. (1884). La Provincia di Lecce. Bozzetti di viaggio, Galatina.

Hartmann, L.M.; Ewald, P. (1957). Gregorii I papae Registrum epistolarum, tom. II.

Houben, H. (1996). Mezzogiorno normanno-svevo, Napoli.

Guidoni. (1991). Storia dell'urbanistica: il Medioevo, secoli VI-XII, Roma.

Licinio, R. (2000). "Dalla «licentia castrum ruinandi» alle disposizioni «castra munienda». Castelli regi e castelli baronali nella Puglia aragonese", in Fonseca, C.D.; Sivo, V. eds., Studi in onore di Giosuè Musca, Bari, pp. 297329.

Licinio, R. (2010). Castelli medievali. Puglia e Basilicata: dai Normanni a Federico II e Carlo I d'Angiò, Bari.

Mauro, A. (1998). Le fortificazioni del Regno di Napoli. Note storiche, Napoli.

Pascalis, G. De. (2004). "Francesco di Giorgio e l'architettura militare in area pugliese", in Nazzaro, B.; Villa, G. eds., Francesco di Giorgio Martini. Rocche, città, paesaggi, Roma, pp. 161-172.

Petrignani, A. (1959). "Gli architetti militari in Puglia", in Atti del IX Congresso Nazionale di Storia dell'Architettura. Bari, 10-16 ottobre 1955, pp. 127-148.

Politano, A. (s.d.). Gallipoli in nove mappe antiche, in http://www.fondazioneterradotranto.it/2015/11/14/gallipoli-innove-mappe-antiche/.

Poso, C.D. (1988), Il salento normanno: territorio, istituzioni, società, Galatina.

Quarta, A. (2016). Il castello di Gallipoli: analisi storica e architettonica, Tesis Doctoral, Università del Salento, Scuola di Specializzazione in beni archeologici.

Quarta, A. (2017a). "The castle of Gallipoli in the defensive system of the Ionian Coast", in Conservaton et valoritation du patrimoine architectural et paysagé des sites côtiers méditerranéens. Résumés de le Conferénce RIPAM 2017. Gênes - 20-22 septembre 2017, Milano, p. 149.

Quarta, A. (2017b). "Il Torrione Poligonale e lo sviluppo della porzione orientale del Castello di Gallipoli", in Defensive architecture of the Mediterranean. XV to XVIII centuries, Publications Universitat d'Alacant, vol. VI, pp. 91-98.

Saladini. C.M. (1987). "Gallipoli”, in Zeri, F. ed., Storia dell'arte italiana, inchiesta sui centri minori, Torino, vol. VIII.

Schettini, F. (1959). "I castelli angioino-aragonesi in Puglia", in Atti del IX Congresso Nazionale di Storia dell'Architettura. Bari, 10-16 ottobre 1955, pp. 117-125.

Sthamer, E. (1995). L'amministrazione dei castelli nel Regno di Sicilia sotto Federico II e Carlo d'Angiò, Bari.

Trinchera, F. (1865). Syllabus Graecarum Membranarum, Napoli.

Vita, R. De. (1974). Castelli, torri ed opere fortificate di Puglia, Bari.

Willemsen, C.A.; Odenthal, D. (1966). Puglia. Terra dei Normanni e degli Svevi, Bari. 